Huijuan Xiu, Rui Cheng, Jinbao Li*, Feiyan Ma, Te Song, Xue Yang, Pan Feng, Xuefei Zhang, Evguenii Kozliak and Yun Ji*

\title{
Effects of acid hydrolysis waste liquid recycle on preparation of microcrystalline cellulose
}

https://doi.org/10.1515/gps-2019-0002

Received April 25, 2018; accepted September 13, 2018.

Abstract: Large amounts of acidic waste are produced on the industrial scale during hydrolysis of partially amorphous cellulose to produce microcrystalline cellulose (MCC). The essential disposal and treatment of this highly acidic liquid wastes the acid feedstock and increases the production cost. To maximize the use of acid without sacrificing the MCC product quality, this project reports a successful attempt to recycle the acid hydrolysis waste liquid, focusing on the impact of waste recycling on MCC morphology and reducing sugar in the hydrolysate. The results showed that when the waste liquid is recycled 1-5 times, no metal accumulation occurred while cellulose particles remained intact, maintaining their shape and size. Their extent of crystallinity remained nearly constant, even increasing slightly with up to three cycles. The concentration of reducing sugar showed growth when recycling the waste liquid up to three times, although not quite to the levels that would allow for its cost-effective fermentation. The acid

\footnotetext{
* Corresponding authors: Jinbao Li, National Experimental Teaching Demonstration Center for Light Chemical Engineering, Shaanxi University of Science \& Technology, Xi'an 710021, China; Shaanxi Province Key Lab of Papermaking Technology and Specialty Paper, Xi'an 710021, China, e-mail: ljb952@163.com and Yun Ji, Department of Chemical Engineering, University of North Dakota, Grand Forks, ND 58202, USA, e-mail: yun.ji@engr.und.edu
}

Huijuan Xiu, National Experimental Teaching Demonstration Center for Light Chemical Engineering, Shaanxi University of Science \& Technology, Xi'an 710021, China; Key Laboratory Pulp and Paper Science \&Technology of Ministry of Education of China, Qilu University of Technology, Jinan 250353, China

Rui Cheng, Feiyan Ma, Te Song, Xue Yang, Xue Yang and Pan Feng, National Experimental Teaching Demonstration Center for Light Chemical Engineering, Shaanxi University of Science \& Technology, Xi'an 710021, China

Xuefei Zhang and Yun Ji, Department of Chemical Engineering, University of North Dakota, Grand Forks, ND 58202, USA Evguenii Kozliak, Department of Chemistry, University of North Dakota, Grand Forks, ND 58202, USA amount to be added at the start of each cycle was near $50 \%$ of that used on the first stage.

Keywords: acid hydrolysis; waste liquid; recycling; microcrystalline cellulose

\section{Introduction}

Microcrystalline cellulose (MCC) is an important plant based powder material, which is produced from biomass by dilute acid hydrolysis. It has been widely used in medicine, food, cosmetic, leather and many other industries as functional additives because of its high crystallinity, high flowability and low water solubility [1-4]. MCC is a purified partially de-polymerized crystalline polymer. Acidic treatment of purified cellulose under well-specified conditions hydrolyzes only the amorphous areas of this polysaccharide while the crystalline domains remain intact [5]. However, a large amount of acidic waste is produced during the MCC production process by acid hydrolysis method. The acid hydrolysis step produces large amounts of waste liquid, which contains mainly the added acid, along with reducing sugars and other cellulose degradation components. A direct discharge of this waste liquid to the environment would result in water and soil pollution, primarily due to high acidity. Thus, industrial production lines typically use $\mathrm{NaOH}$ or $\mathrm{Ca}(\mathrm{OH})_{2}$ to neutralize the waste stream, then transferring it to a wastewater treatment facility. This treatment wastes significant amounts of acid and, at the same time, discards potentially useful organic compounds, as the Chemical Oxygen Demand (COD) content of such wastewater remains high after the acid neutralization [6,7].

If the waste liquid could be recycled, not only the environmental impact but also acid usage and neutralization costs would be reduced, with potential utilization of the accumulated sugar. Sarma et al. applied a combination of membrane technology and activated carbon (AC) adsorption to remove both reducing sugars and carbohydrate oligomers 
from waste liquid followed by a re-use of the resulting sulfuric acid containing liquid [8]. Strathmann proposed a partial acid recovery with a cation selective membrane after hydrolysis of lignocellulose with sulfuric acid, while the other part was converted to $\mathrm{SO}_{\mathrm{x}}$ gas and subsequently to sulfuric acid. However, membrane based technologies, though featuring effective acid separation, require significant investments and logistical support $[9,10]$.

It is of note that the acid hydrolysis waste liquid obtained as a result of MCC preparation has a relatively high acid and low organic solid content. Thus, we postulated that direct recycling of the waste liquid can be applied to re-use the acid, without a damaging effect of by-products, as long as the number of cycles is limited. However, no direct recycling of waste liquid during MCC production has been reported to date, apparently due to the well-known side reactions caused by products of cellulose degradation.

Therefore in this project we have tested this hypothesis by recycling the waste liquid several times in a lab-scale reactor while adding only a fraction of fresh acid at the start of each cycle except for the starting stage. The effects of the number of acid hydrolysis waste liquid cycles were assessed on cellulose microfibril morphology, particle size distribution, crystallinity, yield and thermogravimetric curves. At the same time, changes in the reducing sugar concentration in waste liquor were monitored to explore whether this potential value-added product could be accumulated and used for ethanol production via fermentation.

\section{Materials and methods}

\subsection{Raw materials and chemicals}

Eucalyptus dissolved pulp was provided by Shandong Bapubeibo Pulp and Paper Co., Ltd (Shandong, China). Please not the raw materials for preparation of MCC are not limited to the dissolving pulp. Dissolving pulp was used in this study and its technical quality parameters are shown in Table 1.

37\% concentrated hydrochloric acid was purchased from FuChen Chemical Reagent Factory (Tianjin, China). The copper ethylenediamine solution was purchased from China National Pulp and Paper Research Institute (Beijing, China). All the chemicals were of analytical grade.

\subsection{Preparation of acid hydrolyzed cellulose using recycled waste liquid}

Dissolved pulp made of Eucalyptus was hydrolyzed using either fresh hydrochloric acid or hydrolysis waste liquid, which was circulated for 1 to 5 times. The acid hydrolysis experimental conditions were set up based on our previous research. $50 \mathrm{~g}$ of Eucalyptus dissolved pulp (oven dried weight) in $750 \mathrm{~mL}$ of a $2.5 \mathrm{M}$ hydrochloric acid solution were placed into a KQ2200 ultrasonic cleaner (Kunshan City, China Ultrasound Instrument Co., Ltd.) with the ultrasonic power set at $80 \mathrm{~W}$. Hydrolyzed cellulose was obtained by sonicating the reaction mixture at $80^{\circ} \mathrm{C}$ for $50 \mathrm{~min}$, then the mixture was filtered and separated to obtain the liquid acid waste and solid hydrolyzed cellulose. Solid hydrolyzed cellulose was washed using deionized water to neutral $\mathrm{pH}$ and stored for further experiments. The entire amount of acid waste liquid was recycled for subsequent cellulose acid hydrolysis experiments, with the addition of some fresh acid to make up the acid loss during reaction. The yield of acid hydrolyzed cellulose was calculated by Eq. 1:

Yield $=\frac{\text { Dry weight of hydrolyzed cellulose }(\mathrm{g})}{\text { Dry weight of cellulose raw material }(\mathrm{g})} \times 100 \%$

\subsection{Analysis and characterization of acid hydrolyzed cellulose}

A HITACHI S-4800-type cold field emission scanning electron microscopy - energy dispersive spectrometer (Japan Hitachi) was used to measure the morphology and residual metal content of the hydrolyzed cellulose. The average particle size and particle size distribution of the hydrolyzed cellulose were analyzed using a Mastersizer 2000 laser particle size analyzer (Malvern Instruments Ltd., UK). The thermal stability of hydrolyzed cellulose was measured by a Bruker STA449F3 TG-DSC synchronous thermal analyzer (NETZSCH, Germany). The scanning temperature range was $35 \sim 700^{\circ} \mathrm{C}$ and nitrogen was used as an inert gas. The heating rate was $10^{\circ} \mathrm{C} / \mathrm{min}$.

The crystallinity of hydrolyzed cellulose was measured using a D/max 2200PC X-ray diffractometer (XRD) (Rigaku, Japan). The samples were freeze-dried at $-50^{\circ} \mathrm{C}$ for $24 \mathrm{~h}$. The crystallinity test conditions were as follows: the maximum

Table 1: Eucalyptus dissolved pulp quality parameters.

\begin{tabular}{ccccc}
\hline $\boldsymbol{\alpha}$-cellulose content \% & Degree of polymerization & Degree of crystallinity \% & Whiteness \% ISO & Ash content \% \\
\hline 92.66 & 661 & 58.92 & 86.3 & 0.08 \\
\hline
\end{tabular}


tube voltage $60 \mathrm{kV}$, maximum tube current $80 \mathrm{~mA}, \mathrm{Cu} \mathrm{Ka}$ radiation $(\lambda=0.1518 \mathrm{~nm})$, Ni filter, diffraction angle $2 \theta$ scan range $6-50^{\circ}$, scanning speed $2^{\circ} / \mathrm{min}$. The sample crystallinity was calculated according to Eq. 2 [11-13].

$$
\text { Crystallinity }=\frac{I_{002}-I_{A m}}{I_{002}} \times 100 \%
$$

where $\mathrm{I}_{002}$ is the diffraction intensity at a diffraction angle of $2 \theta=22.5^{\circ}$ and $\mathrm{I}_{\mathrm{Am}}$ is the diffraction intensity at a diffraction angle of $2 \theta=18.0^{\circ}$.

\subsection{Sugar, acid and metal content analysis in waste liquid}

The reducing sugar content in the acid waste liquid was measured by the DNS method using a Model $722 \mathrm{~N}$ visible spectrophotometer (Shanghai Jingke Instrument Co., Ltd., China) [14]. The metal ion concentrations was determined by the analysis of EDS spectra. The amount of acid was assessed by titration. The amount of fresh acid to be added
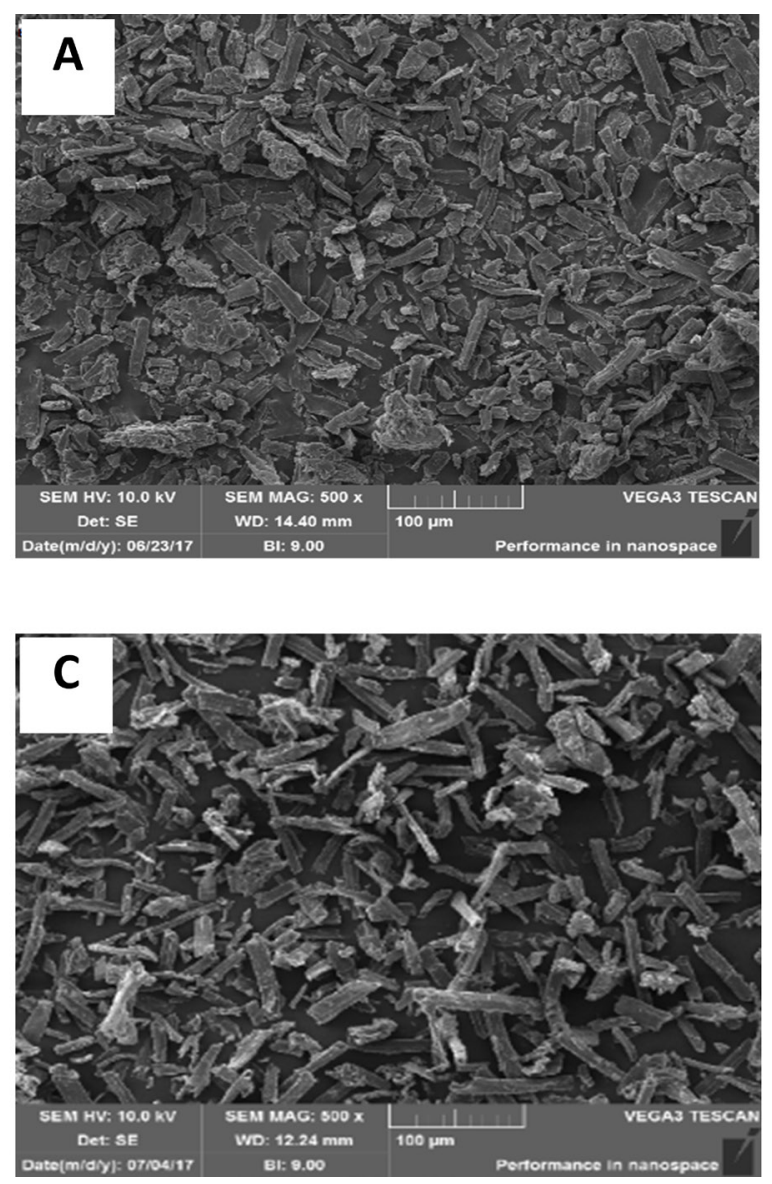

at the start of each cycle was calculated as the difference between the initial and remaining acid amounts.

The error bars shown in Figures represent one standard deviation, indicating our experimental data have good repeatability.

\section{Results and discussion}

\subsection{Microscopic morphology of hydrolyzed cellulose after several waste liquid cycles}

Figure 1 shows the SEM images of hydrolyzed cellulose after several waste liquid cycles. As can be seen in Figures 1a-d, the hydrolyzed cellulose morphology was mainly represented by short rod-shaped particles, similar to the data reported earlier $[15,16]$. Only relatively small changes were detected between different samples. For cellulose obtained by a single passage hydrolysis
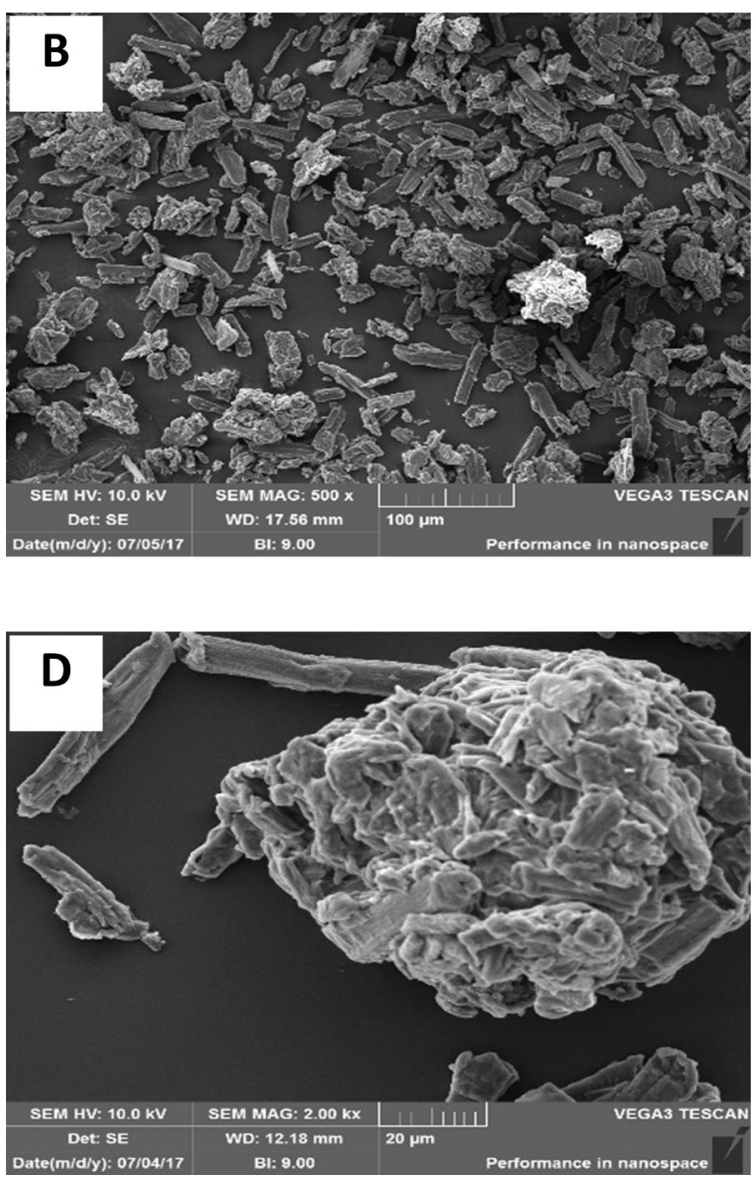

Figure 1: SEM image of hydrolyzed cellulose under different waste liquid cycles (a) uncycled (single passage), (b) recycled three times, (c) recycled five times, (d) an example of an agglomerated floccule. 
(uncycled), particles appeared slightly smaller (Figure 1a). After several cycles, the particle distribution became more uniform (Figure 1c). Perhaps, the most conspicuous change was observed concerning the agglomerated floccules, which appeared in all hydrolyzed cellulose products (Figure 1d). The number of such floccules consistently decreased with waste liquid recycling, becoming significantly less abundant after five cycles.

\subsection{Effects of the number of waste liquid cycles on the average particle size and particle size distribution of hydrolyzed cellulose}

The obtained particle size distribution profiles are shown in Figure 2. The average particle sizes of all hydrolyzed cellulose samples, both without recycling and with a varied number of cycles, were similar, with a maximum near $27 \mu \mathrm{m}$, thus corroborating the microscopic observations. The particle size distribution of hydrolyzed cellulose showed polydispersity, with most of the particles being within a range of 15 to $\sim 50 \mu \mathrm{m}$. The difference in the size distribution between the recycled and non-recycled cellulose was not significant.

\subsection{Effect of waste cycles on the crystallinity of hydrolyzed cellulose}

Figure 3 shows the X-ray diffraction pattern of hydrolyzed cellulose that underwent a varied number of waste liquid cycles. The diffraction peaks at diffraction angles $(2 \theta)$ of $15.3^{\circ}, 18.2^{\circ}, 22.8^{\circ}$ and $34.7^{\circ}$ correspond to the $101,101,002$

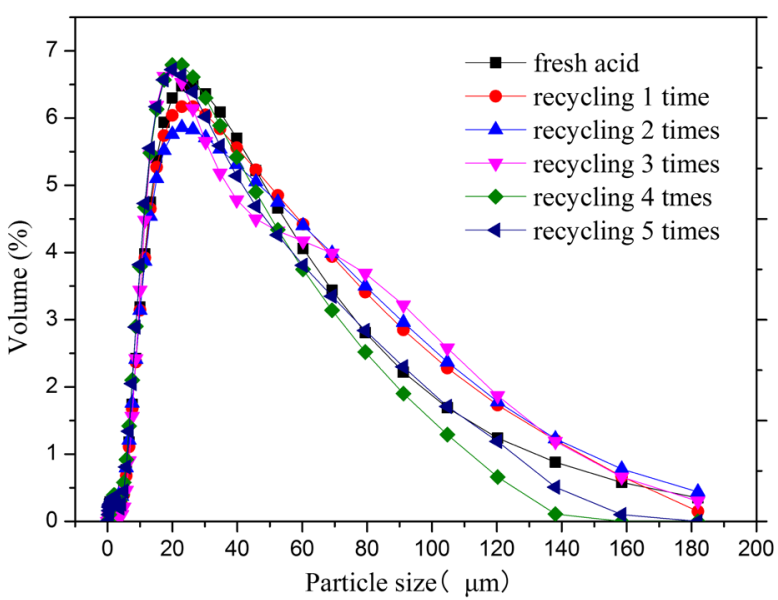

Figure 2: Distribution of cellulose particle size under different waste liquid cycles. and 040 planes of cellulose crystals, respectively, and so these crystals can be assigned to typical cellulose type I [1721], having better mechanical properties than other cellulose types [22]. The pulp raw material featured the same peak positions as those of the original cellulose, regardless of the number of waste liquid cycles, indicating that the crystal structure of cellulose was not altered after acid hydrolysis, with the number of waste liquid cycles having no effect.

Yet, the diffraction peaks at both the 101 and 002 crystal planes of the hydrolyzed cellulose obtained from the recycled waste liquid samples were stronger than those of both the raw material and the hydrolyzed cellulose treated only once, with fresh acid. The calculated degree of crystallinity of hydrolyzed cellulose based on the XRD patterns is shown in Figure 4, indicating an increase in cellulose crystallinity.

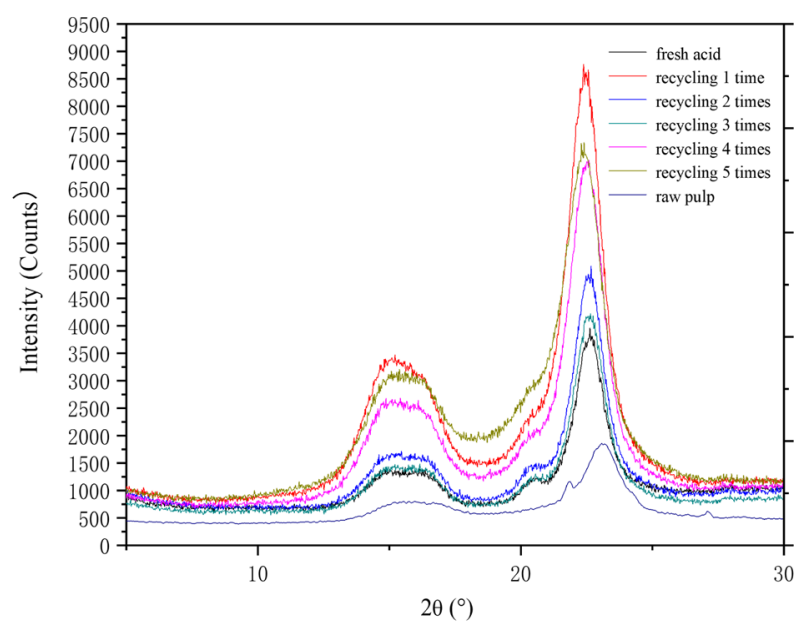

Figure 3: XRD patterns of the raw material and hydrolyzed cellulose under a varied number of waste cycles.

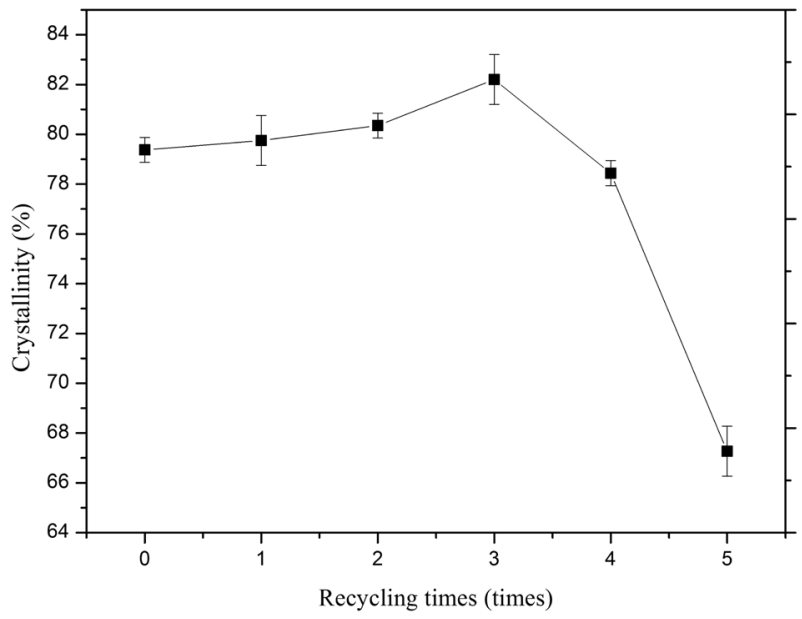

Figure 4: Changes in the extent of crystallinity of hydrolyzed cellulose under a varied number of waste liquid cycles. 
This qualitative observation was confirmed with quantitative measurements. The degree of crystallinity increased slightly yet steadily with the number of waste liquid cycles and reached the highest value of $82.2 \%$ when the waste liquid was recycled three times, while it was $79.4 \%$ when hydrolyzed cellulose was prepared with fresh acid.

However, when the number of cycles continued to increase further, the extent of crystallinity declined. After the fifth cycle, the crystallinity of hydrolyzed cellulose dropped sharply to $67.3 \%$; this value failed to meet the requirement for MCC high crystallinity. Therefore to ensure the high quality of the final product, MCC, the waste liquid should be recycled only up to three times.

\subsection{Effect of the number of waste liquid cycles on the reducing sugar content}

To determine whether the reducing sugars produced by cellulose degradation during acid hydrolysis could accumulate in the waste liquid upon its recycling, we measured the changes occurring in the reducing sugar content in waste liquid after each cycle, as shown in Figure 5. The reducing sugarconcentration in the unrecycled waste liquid was $1.08 \mathrm{~g} / \mathrm{L}$, and then it increased with the increase of the number of waste liquid cycles, reaching a peak value of $3.32 \mathrm{~g} / \mathrm{L}$ after the third cycle. Following this point, the reducing sugar concentration decreased sharply and then remained low, presumably due to its degradation. Literature shows that the reducing sugar in waste liquids could be further degraded into organic acids, 5-hydroxymethyl furfural and other by-products [23,24].

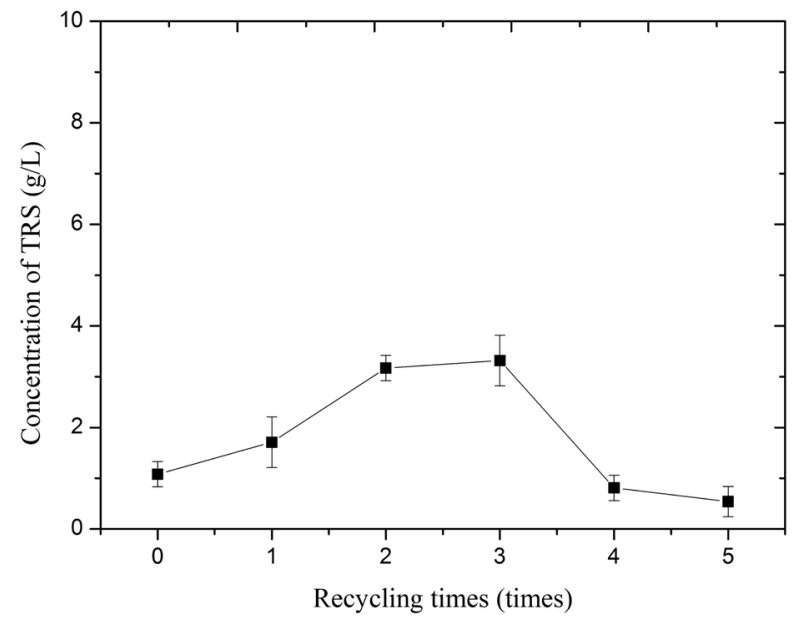

Figure 5: Reducing sugar concentrations in waste liquid after a varied number of cycles.
The maximum observed reducing sugar concentration, $3.32 \mathrm{~g} / \mathrm{L}$, was much lower than that of $15-25 \mathrm{~g} / \mathrm{L}$ required for ethanol production after conventional cellulose hydrolysis $[25,26]$. Thus, the use of accumulated reducing sugar for ethanol production is technically feasible, but not economically favorable.

\subsection{Effect of waste liquid recycling on the residual metal content in hydrolyzed cellulose}

MCC has strict requirements on the heavy metal content, which should not exceed $10 \mathrm{ppm}$ (Pharmacopoeia of the People's Republic of China, 2015). Figure 6 shows the metal ion concentrations in acidic waste liquid after three cycles. As can be seen, no heavy metals were detected in the hydrolyzed cellulose sample except for $\mathrm{Au}$, only because the surface was sprayed with gold prior to the test.

\subsection{Effects of waste liquid recycling on the yield of hydrolyzed cellulose}

The yields of hydrolyzed cellulose after a varied number of waste liquid cycles are shown in Figure 7. With the increase in the number of waste liquid cycles, the yield of hydrolyzed cellulose first decreased slightly, from $92.3 \%$ to $88.7 \%$, then increased to $91.2 \%$. Thus, the waste liquid recycling had little effect on the main product yield.

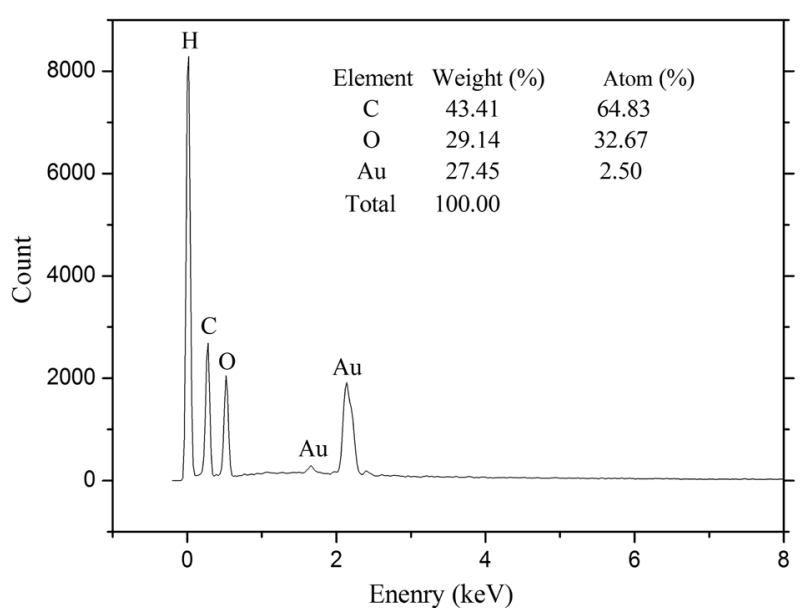

Figure 6: EDS spectra of hydrolyzed cellulose after three waste liquid cycles. 


\subsection{Effect of waste liquid recycling on thermal stability of hydrolyzed cellulose}

Figure 8 shows the TG curves of the resulting hydrolyzed cellulose after a varied number of waste liquid cycles. They all turned out to be near identical to the curve of the product obtained with fresh acid, indicating that the waste liquid recycling had no effect on the thermal stability of hydrolyzed cellulose. This information is consistent with literature stating that the thermostability of hydrolyzed cellulose is similar to that of natural cellulose, with the main change occurring at $240-400^{\circ} \mathrm{C}$, when pyrolytic low-molecular-weight volatile compounds evolve $[27,28]$.

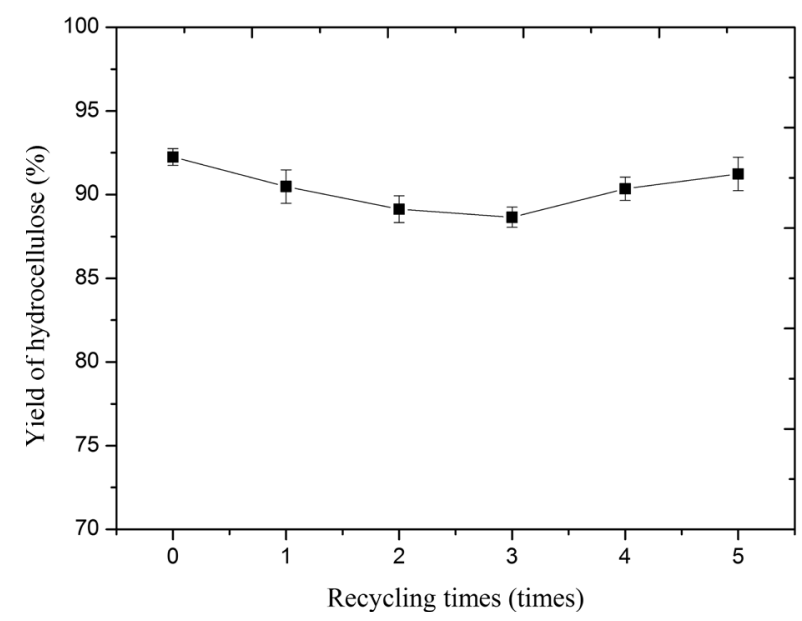

Figure 7: Yield of hydrocellulose after several waste liquid cycles.

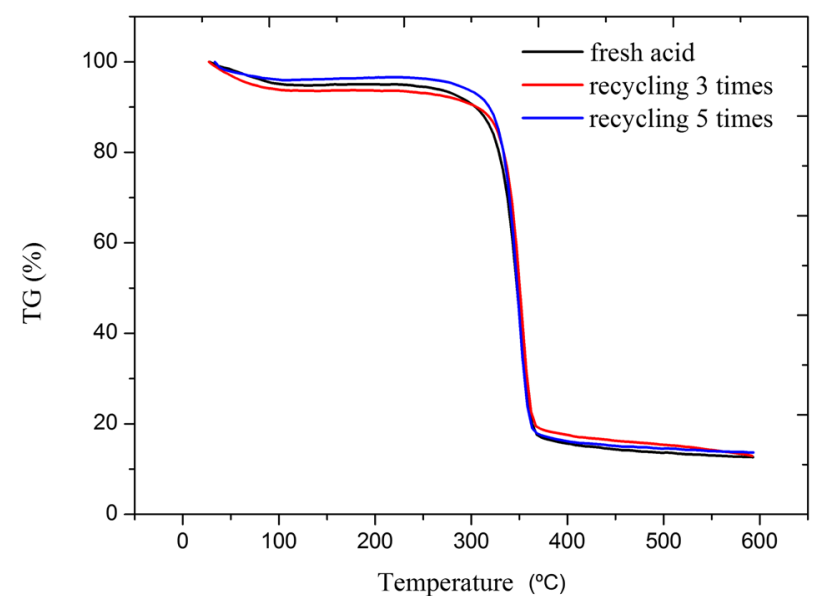

Figure 8: Thermogravimetric (TG) curves of hydrolyzed cellulose after a varied number of waste liquid cycles.

\subsection{Fresh acid usage}

Figure 9 shows the amounts of fresh acid required for hydrolysis after a varied number of waste liquid cycles, to match the original acid concentration. As expected, the recycling of waste liquor significantly reduced the amount of fresh acid, asit was used up for hydrolysis. Yet, when the waste liquid was recycled once, twice and three times, the amounts of fresh acid required for acid hydrolysis were only $62.1 \%$, $50.0 \%$ and $43.4 \%$ of the fresh acid amount, respectively. Therefore, waste liquid recycling can significantly reduce the production cost of hydrolyzed cellulose while also reducing the amount of waste liquid and waste water discharge, thus benefiting the environment. As shown above, this can be done without sacrificing the product quality, as long as the number of cycles does not exceed three.

\section{Conclusion}

Waste liquid recycling was shown to be feasible in the process of cellulose acid hydrolysis. With up to three cycles, the quality of hydrolyzed cellulose remained high. Moreover, the degree of crystallinity of hydrolyzed cellulose even increased slightly from $79.4 \%$ to $82.2 \%$. The reducing sugar concentration in the waste liquid accumulated concomitantly, reaching the maximum value of $3.32 \mathrm{~g} / \mathrm{L}$ after three waste liquid cycles, although the ethanol production was still not economically feasible with this low concentration. However, waste liquid recycling can significantly reduce the amount of fresh acid usage and waste disposal, significantly reducing the MCC production cost and contributing to environmental protection.

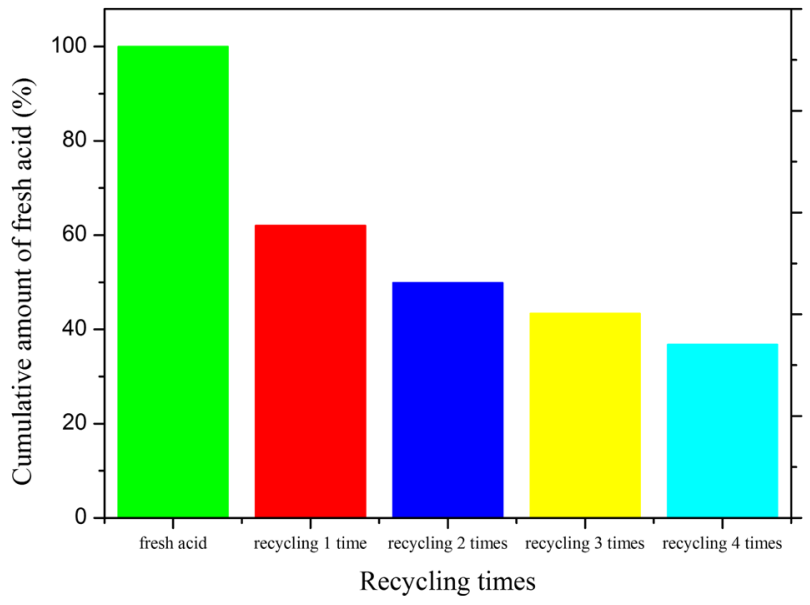

Figure 9: Fresh acid required for acid hydrolysis after a varied number of waste liquid cycles. 
Acknowledgements: This research project was supported by the National Key Research and Development Plan of China (2017YFB0307901), Key Research and Development Projects of Shaanxi Province (2017ZDXM-SF-090), Research Planning Program of Key Laboratory Pulp and Paper Science \&Technology of Ministry of Education of China (08031352), the Doctoral Scientific Research Foundation (Grant No. BJ13-14) of Shaanxi University of Science and Technology, and Research Plan Project (Project \# 15JK1101) of Education Department of Shaanxi Province in China.

\section{References}

[1] Siró I., Plackett D., Microfibrillated cellulose and new nanocomposite materials: A review. Cellulose, 2010, 17, 459-494.

[2] Nada A.A.M.A., El-Kady M.Y., Abd El-Sayed E.S., Amine F.M., Preparation and characterization of microcrystalline cellulose (MCC). BioResources, 2009, 4, 1359-1371.

[3] Chuayjuljit S., Su-Uthai S., Charuchinda S., Poly(vinyl chloride) film filled with microcrystalline cellulose prepared from cotton fabric waste: Properties and biodegradability study. Waste Manag. Res., 2010, 28, 109-117.

[4] El-Sakhawy M., Hassan M.L., Physical and mechanical properties of microcrystalline cellulose prepared from agricultural residues. Carbohydr. Polym., 2007, 67, 1-10.

[5] Nguyen X.T., Process for preparing microcrystalline cellulose, U.S. patent 700,551,4B2, 2002-10-16.

[6] Russell D.L., Practical Wastewater Treatment. John Wiley \& Sons, 2006.

[7] Tchobanoglus G., Burton F., Stensel H.D., Wastewater engineering: treatment and reuse. American Water Works Association, 2003, 95(5), 201.

[8] Sarma S.J., Ayadi M., Brar S.K., Berry R., Sustainable commercial nanocrystalline cellulose manufacturing process with acid recycling. Carbohydr. Polym., 2017, 156, 26-33.

[9] Strathmann H., Membrane Separation Processes in Advanced Waste Water Treatment. Pure Appl. Chem., 1976, 46, 213-220.

[10] Strathmann H., Membrane separation processes. J. Memb. Sci., 1981, 9, 121-189.

[11] Segal L., Creely J.J., Martin A.E., Conrad C.M., An Empirical Method for Estimating the Degree of Crystallinity of Native Cellulose Using the X-Ray Diffractometer. Text Res. J., 1959, 29, 786-794.

[12] Ling Z., Chen S., Zhang X., Xu F., Exploring crystalline-structural variations of cellulose during alkaline pretreatment for enhanced enzymatic hydrolysis. Bioresour. Technol., 2017, 224, 611-617.

[13] Choi K.H., Kim A.R., Cho B.U., Effects of alkali swelling and beating treatments on properties of kraft pulp fibers. BioResources, 2016, 11, 3769-3782.
[14] Miller G.L., Use of Dinitrosalicylic Acid Reagent for Determination of Reducing Sugar. Anal. Chem., 1959, 31, 426-428.

[15] Kalita R.D., Nath Y., Ochubiojo M.E., Buragohain A.K., Extraction and characterization of microcrystalline cellulose from fodder grass; Setaria glauca (L) P. Beauv, and its potential as a drug delivery vehicle for isoniazid, a first line antituberculosis drug. Colloids Surfaces B. Biointerfaces, 2013, 108, 85-89.

[16] Adel A.M., Abd El-Wahab Z.H., Ibrahim A.A., Al-Shemy M.T., Characterization of microcrystalline cellulose prepared from lignocellulosic materials. Part II: Physicochemical properties. Carbohydr. Polym., 2011, 83, 676-687.

[17] Shimizu K.I, Furukawa H., Kobayashi N., Itaya Y., Satsuma A., Effects of Brønsted and Lewis acidities on activity and selectivity of heteropolyacid-based catalysts for hydrolysis of cellobiose and cellulose. Green Chem., 2009, 11, 1627-1632.

[18] Lu Q., Tang L., Lin F., Wang S., Chen Y., Chen X., et al., Preparation and characterization of cellulose nanocrystals via ultrasonication-assisted $\mathrm{FeCl}_{3}$-catalyzed hydrolysis. Cellulose, 2014, 21, 3497-3506.

[19] Jahan M.S., Saeed A., He Z., Ni Y., Jute as raw material for the preparation of microcrystalline cellulose. Cellulose, 2011, 18, 451-459.

[20] Ciolacu D., On the supramolecular structure of cellulose allomorphs after enzymatic degradation. J. Optoelectron. Adv. Mat., 2007 , 9(4), 1033-1037.

[21] French A.D., Idealized powder diffraction patterns for cellulose polymorphs. Cellulose, 2014, 21, 885-896.

[22] Morán J.I., Alvarez V.A., Cyras V.P., Vázquez A., Extraction of cellulose and preparation of nanocellulose from sisal fibers. Cellulose, 2008, 15, 149-159.

[23] Girisuta B., Janssen L.P.B.M., Heeres H.J., Kinetic study on the acid-catalyzed hydrolysis of cellulose to levulinic acid. Ind. Eng. Chem. Res., 2007, 46, 1696-1708.

[24] Modig T., Almeida J.R.M., Gorwa-Grauslund M.F., Lidén G., Variability of the response of Saccharomyces cerevisiae strains to lignocellulose hydrolysate. Biotechnol. Bioeng., 2008, 100, 423-429.

[25] Palmqvist E., Hahn-Hägerdal B., Fermentation of lignocellulosic hydrolysates. I: Inhibition and detoxification. Bioresour. Technol., 2000, 74, 17-24.

[26] Palmqvist E., Hahn-Hägerdal B., Fermentation of lignocellulosic hydrolysates. II: Inhibitors and mechanisms of inhibition. Bioresour. Technol., 2000, 74, 25-33.

[27] Kuo C.H., Lee C.K., Enhancement of enzymatic saccharification of cellulose by cellulose dissolution pretreatments. Carbohydr. Polym., 2009, 77, 41-46.

[28] Cui T., Li J., Yan Z., Yu M., Li S., The correlation between the enzymatic saccharification and the multidimensional structure of cellulose changed by different pretreatments. Biotechnol. Biofuels, 2014, 7(1), 134. 